\title{
Expansivity and shadowing in linear dynamics
}

\author{
Nilson C. Bernardes Jr. ${ }^{a, 1}$, Patricia R. Cirilo ${ }^{\mathrm{b}, 2}$, Udayan B. Darji ${ }^{\mathrm{c}, \mathrm{d}}$, \\ Ali Messaoudi ${ }^{\mathrm{e}, 3}$, Enrique R. Pujals ${ }^{\mathrm{f}, *, 4}$ \\ a Departamento de Matemática Aplicada, Instituto de Matemática, Universidade Federal do Rio de \\ Janeiro, Caixa Postal 68530, Rio de Janeiro, RJ, 21945-970, Brazil \\ b Instituto de Ciência e Tecnologia, Universidade Federal de São Paulo, Avenida Cesare Mansueto Giulio \\ Lattes, $n^{\circ}$ 1201, São José dos Campos, SP, 12247-014, Brazil \\ c Department of Mathematics, University of Louisville, Louisville, KY 40292, USA \\ d Department of Mathematics, Ashoka University, Rajiv Gandhi Education City Kundli, Rai 131029, India \\ e Departamento de Matemática, Universidade Estadual Paulista, Rua Cristóvão Colombo, 2265, \\ Jardim Nazareth, São José do Rio Preto, SP, 15054-000, Brazil \\ ${ }_{\mathrm{f}}$ Instituto Nacional de Matemática Pura e Aplicada, Estrada Dona Castorina 110, Rio de Janeiro, RJ, \\ 22460-320, Brazil
}

\section{A R T I C L E I N F O}

\section{Article history:}

Received 28 August 2017

Available online 19 December 2017

Submitted by J. Bonet

\section{Keywords:}

Expansive

Hypercyclic

Li-Yorke

Hyperbolic

Shadowing

Weighted shifts

\begin{abstract}
A B S T R A C T
In the early 1970's Eisenberg and Hedlund investigated relationships between expansivity and spectrum of operators on Banach spaces. In this paper we establish relationships between notions of expansivity and hypercyclicity, supercyclicity, Li-Yorke chaos and shadowing. In the case that the Banach space is $c_{0}$ or $\ell_{p}(1 \leq p<\infty)$, we give complete characterizations of weighted shifts which satisfy various notions of expansivity. We also establish new relationships between notions of expansivity and spectrum. Moreover, we study various notions of shadowing for operators on Banach spaces. In particular, we solve a basic problem in linear dynamics by proving the existence of nonhyperbolic invertible operators with the shadowing property. This contrasts with the expected results for nonlinear dynamics on compact manifolds, illuminating the richness of dynamics of infinite dimensional linear operators.
\end{abstract}

() 2017 Elsevier Inc. All rights reserved.

\section{Introduction}

The study of the dynamics of continuous linear operators on infinite dimensional Banach (or Fréchet) spaces has witnessed a great development during the last three decades and many links between this area

\footnotetext{
* Corresponding author.

E-mail addresses: bernardes@im.ufrj.br (N.C. Bernardes), pcirilo@unifesp.br (P.R. Cirilo), ubdarj01@louisville.edu

(U.B. Darji), messaoud@ibilce.unesp.br (A. Messaoudi), enrique@impa.br (E.R. Pujals).

1 Partially supported by CNPq.

2 Partially supported by FAPESP \#2011/11663-5.

3 Partially supported by CNPq and by FAPESP \#2013/24541-0.

4 Partially supported by CNPq.
} 
and other areas of mathematics, such as ergodic theory, number theory and geometry of Banach spaces, have been established. We refer the reader to the books [3,18] and to the more recent papers $[4,7-9,17]$, where many additional references can be found. On the other hand, the notions of expansivity and shadowing play important roles in many branches of the area of dynamical systems, including topological dynamics, differentiable dynamics and ergodic theory; see $[1,23,24,30]$, for instance. Our goal in this paper is to investigate the notions of expansivity and shadowing in the context of linear dynamics, thereby complementing previous works by various authors. In particular, we give a class of examples of operators exhibiting the shadowing property which are not hyperbolic, however they are chaotic. Such examples show the richness of linear dynamics and its difference from finite dimensional nonlinear dynamics, yielding counterintuitive results to the corresponding ones from finite dimensional smooth dynamics.

Let us now describe the organization of the article.

In Section 2 we fix the notations and recall the definitions and a few results which will be important in our work.

In Section 3 we investigate the notions of shadowing, limit shadowing and $\ell_{p}$ shadowing for invertible operators on Banach spaces. It is well-known that invertible hyperbolic operators have the shadowing property and that the converse holds for invertible operators on finite dimensional spaces [22] and for invertible normal operators on Hilbert spaces [21]. Moreover, the converse also holds for certain sequences of finite dimensional operators considered in [25]. This implies that for $C^{1}$ diffeomorphisms of $m$-dimensional closed smooth manifolds, hyperbolicity is equivalent to Lipschitz shadowing [25]. A basic open problem in linear dynamics is whether the shadowing property implies hyperbolicity for invertible operators on Banach (or Hilbert) spaces. This problem appeared explicitly in [21, Page 148], for instance. In Theorem B, we solve this problem in the negative by proving the existence of operators with the shadowing property that exhibit several types of chaotic behaviors (they are simultaneously frequently hypercyclic, Devaney chaotic, mixing and densely distributionally chaotic) and, in particular, are not even expansive. We also establish a useful sufficient condition for shadowing (Theorem A) and consider the case of noninvertible operators by looking at the notion of positive shadowing.

In Section 4 we investigate various notions of expansivity for operators on Banach spaces. We establish relationships between these notions and some popular notions in linear dynamics, namely: hypercyclicity, supercyclicity and Li-Yorke chaos. In particular, we prove that a uniformly expansive operator cannot be Li-Yorke chaotic and hence it cannot be hypercyclic (Theorem C), but we observe that every infinitedimensional separable Banach space supports a supercyclic uniformly expansive operator. On the other hand, we prove that a hyperbolic operator with nontrivial hyperbolic splitting cannot be supercyclic. We also investigate the relationship between expansivity of an operator and its spectrum. In particular, we expand earlier results of Eisenberg and Hedlund [15,16,19] and Mazur [21]. In 1966 Eisenberg [15] proved that if $T$ is an invertible operator on $\mathbb{C}^{n}$, then $T$ is expansive if and only if $T$ has no eigenvalue on the unit circle $\mathbb{T}$. Subsequently, Eisenberg and Hedlund [16] and Hedlund [19] studied expansive and uniformly expansive operators on Banach spaces. They showed that an invertible operator $T$ is uniformly expansive if and only if $\sigma_{a}(T)$, the approximate point spectrum of $T$, does not intersect $\mathbb{T}$. As a corollary, they obtained that invertible hyperbolic operators are uniformly expansive. Mazur [21] proved that an invertible normal operator $T$ on a Hilbert space $H$ is expansive if and only if $\sigma_{p}\left(T^{*} T\right)$, the point spectrum of $T^{*} T$, does not intersect $\mathbb{T}$. We show that an invertible operator $T$ is uniformly positively expansive if and only if $\sigma_{a}(T)$ does not intersect the closed unit disc $\overline{\mathbb{D}}$ (Theorem D). Moreover, we expand Mazur's result by giving a necessary and sufficient condition for a normal operator to be positively expansive. Our techniques also yield a simpler proof of his result. We also prove that every expansive operator with the shadowing property is uniformly expansive.

In Section 5 we study weighted shifts. Due to their importance in operator theory and its applications, the dynamics of weighted shifts has received special attention from the specialists in linear dynamics. Many dynamical properties have been extensively studied and, in many cases, complete characterizations 
were obtained. For instance, Salas [29] characterized hypercyclicity and weak mixing whereas Costakis and Sambarino [12] characterized mixing for unilateral and bilateral weighted shifts on $\ell_{2}(\mathbb{N})$ and $\ell_{2}(\mathbb{Z})$, respectively. We obtain here complete characterizations of various notions of expansivity for unilateral and bilateral weighted shifts on the Banach spaces $c_{0}(A)$ and $\ell_{p}(A)(1 \leq p<\infty)$, where $A=\mathbb{N}$ or $\mathbb{Z}$ (Theorem E). As applications we give examples of hypercyclic positively expansive operators and of supercyclic uniformly positively expansive operators.

\section{Preliminaries}

As usual, $\mathbb{N}$ denotes the set of all positive integers and $\mathbb{N}_{0}=\mathbb{N} \cup\{0\}$. Moreover, $\mathbb{D}$ and $\mathbb{T}$ denote the open unit disc and the unit circle in the complex plane $\mathbb{C}$, respectively.

Given a Banach space $X, S_{X}=\{x \in X:\|x\|=1\}$ is the unit sphere of $X$. Moreover, by an operator on $X$ we mean a bounded linear map $T: X \rightarrow X$.

If $T$ is an operator on a complex Banach space $X$, then $\sigma(T), \rho(T), \sigma_{p}(T), \sigma_{r}(T), \sigma_{a}(T)$ and $r(T)$ denote the spectrum, the resolvent set, the point spectrum, the residual spectrum, the approximate point spectrum and the spectral radius of $T$, respectively. In the case $T$ is an operator on a real Banach space $X$, we define $\sigma(T)=\sigma\left(T_{\mathbb{C}}\right), \rho(T)=\rho\left(T_{\mathbb{C}}\right)$, and so on, where $T_{\mathbb{C}}$ denotes the complexification of $T$. The spectral radius formula asserts that $r(T)=\lim _{n \rightarrow \infty}\left\|T^{n}\right\|^{\frac{1}{n}}$. Moreover, if $T$ is a self-adjoint operator on a complex Hilbert space $H$ and $x \in H$, then there is a unique positive Radon measure $\mu$ on $\sigma(T)$ such that

$$
\langle f(T) x, x\rangle=\int_{\sigma(T)} f(t) d \mu(t) \quad \text { for all } f \in C(\sigma(T)) .
$$

So, $\mu(\sigma(T))=\|x\|^{2}$. The measure $\mu$ is called the spectral measure associated to $T$ and $x$. We refer the reader to the books $[14,31]$ for more information concerning spectrum.

Definition 1. An invertible operator $T$ on a Banach space $X$ is said to be expansive (positively expansive) if for every $z \in S_{X}$, there exists $n \in \mathbb{Z}(n \in \mathbb{N})$ such that $\left\|T^{n} z\right\| \geq 2$.

Definition 2. An invertible operator $T$ on a Banach space $X$ is said to be uniformly expansive (uniformly positively expansive) if there exists $n \in \mathbb{N}$ such that

$$
z \in S_{X} \Longrightarrow\left\|T^{n} z\right\| \geq 2 \text { or }\left\|T^{-n} z\right\| \geq 2 \quad\left(z \in S_{X} \Longrightarrow\left\|T^{n} z\right\| \geq 2\right) .
$$

We remark that for the definitions of positive expansivity and uniform positive expansivity, $T$ need not be invertible. Also, one can replace the number 2 in the above definitions by any number $c>1$. Moreover, the above definition of expansivity agrees with the usual one in metric spaces, since it is equivalent to the existence of a constant $e>0$ such that, for any pair $x, y$ of distinct points in $X$, there exists $n \in \mathbb{Z}$ with $\left\|T^{n} x-T^{n} y\right\| \geq e$.

Remark 3. In the case $T$ is an operator on a real Banach space $X$, the (uniform) (positive) expansivity of $T$ is equivalent to the corresponding property for its complexification $T_{\mathbb{C}}$.

Definition 4. An operator $T$ is said to be hyperbolic if $\sigma(T) \cap \mathbb{T}=\emptyset$.

It is classical that $T$ is hyperbolic if and only if there are an equivalent norm $\|\cdot\|$ on $X$ and a splitting $X=X_{s} \oplus X_{u}, T=T_{s} \oplus T_{u}$ (the hyperbolic splitting of $T$ ), where $X_{s}$ and $X_{u}$ are closed $T$-invariant subspaces of $X$ (the stable and the unstable subspaces for $T$ ), $T_{s}=\left.T\right|_{X_{s}}$ is a proper contraction (i.e., 
$\left.\left\|T_{s}\right\|<1\right), T_{u}=\left.T\right|_{X_{u}}$ is invertible and is a proper dilation (i.e., $\left\|T_{u}^{-1}\right\|<1$ ), and the identification of $X$ with the product $X_{s} \times X_{u}$ identifies $\|\cdot\|$ with the max norm on the product.

It is also known [19] that $T$ is uniformly expansive if and only if $\sigma_{a}(T) \cap \mathbb{T}=\emptyset$. Hence, every invertible hyperbolic operator is uniformly expansive.

Given a metric space $M$ and a homeomorphism $h: M \rightarrow M$, recall that $\left(x_{n}\right)_{n \in \mathbb{Z}}$ is a $\delta$-pseudotrajectory of $h(\delta>0)$ if $d\left(h\left(x_{n}\right), x_{n+1}\right) \leq \delta$ for all $n \in \mathbb{Z}$. $h$ has the shadowing property if for every $\varepsilon>0$ there is $\delta>0$ such that every $\delta$-pseudotrajectory $\left(x_{n}\right)_{n \in \mathbb{Z}}$ of $h$ is $\varepsilon$-shadowed by a real trajectory of $h$, that is, there is $x \in M$ such that $d\left(x_{n}, h^{n}(x)\right)<\varepsilon$ for all $n \in \mathbb{Z}$. Moreover, $h$ has the Lipschitz shadowing property if there exists $K>0$ such that $\delta$ can be chosen satisfying that $\varepsilon<K \delta$. More generally, we call it $\alpha$-Hölder shadowing property, $0<\alpha \leq 1$, if $\delta$ can be chosen so that $\varepsilon<K \delta^{\alpha}$.

Remark 5. In the case of operators, it is enough to check the above condition for a single $\varepsilon>0$. More precisely, if $T$ is an invertible operator on a Banach space $X$ and if for some $\varepsilon>0$ there exists $\delta>0$ such that every $\delta$-pseudotrajectory of $T$ is $\varepsilon$-shadowed by a real trajectory of $T$, then $T$ has the shadowing property. Moreover, any operator satisfying the shadowing property trivially satisfies the Lipschitz shadowing property.

We shall also consider here the notions of limit shadowing and $\ell_{p}$ shadowing. The homeomorphism $h$ has the limit shadowing property if for every sequence $\left(x_{n}\right)_{n \in \mathbb{Z}}$ in $M$ with $\lim _{|n| \rightarrow \infty} d\left(h\left(x_{n}\right), x_{n+1}\right)=0$, there exists $x \in M$ such that $\lim _{|n| \rightarrow \infty} d\left(x_{n}, h^{n}(x)\right)=0$. Moreover, $h$ has the $\ell_{p}$ shadowing property $(1 \leq p<\infty)$ if for every sequence $\left(x_{n}\right)_{n \in \mathbb{Z}}$ in $M$ with $\sum_{n \in \mathbb{Z}} d\left(h\left(x_{n}\right), x_{n+1}\right)^{p}<\infty$, there exists $x \in M$ such that $\sum_{n \in \mathbb{Z}} d\left(x_{n}, h^{n}(x)\right)^{p}<\infty$. See [23,24] for more information and additional references on shadowing.

We have the following result, whose simple proof is left to the reader.

Proposition 6. Let $T$ be an invertible operator on a Banach space $X$. Suppose that $X=M \oplus N$, where $M$ and $N$ are closed $T$-invariant subspaces of $X$. Then $T$ has the shadowing property (the limit shadowing property, the $\ell_{p}$ shadowing property) if and only if so do $\left.T\right|_{M}$ and $\left.T\right|_{N}$.

Corollary 7. If $T$ is an invertible operator on a real Banach space $X$, then $T$ has the shadowing property (the limit shadowing property, the $\ell_{p}$ shadowing property) if and only if so does its complexification $T_{\mathbb{C}}$.

Let us also recall that an operator $T$ on a Banach space $X$ is Li-Yorke chaotic if it has an uncountable scrambled set $U$, i.e., for all $x, y \in U$ with $x \neq y$, we have that

$$
\liminf _{n \rightarrow \infty}\left\|T^{n} x-T^{n} y\right\|=0 \quad \text { and } \quad \limsup _{n \rightarrow \infty}\left\|T^{n} x-T^{n} y\right\|>0
$$

We say that $T$ is hypercyclic if it has a dense orbit, i.e., $\left\{T^{n} x: n \geq 0\right\}$ is dense in $X$ for some $x \in X$. Finally, $T$ is supercyclic if there exists $x \in X$ whose projective orbit $\left\{\lambda T^{n} x: n \geq 0, \lambda\right.$ scalar $\}$ is dense in $X$. We refer the reader to the books $[3,18]$.

If $X=\ell_{p}(\mathbb{Z})(1 \leq p<\infty)$ or $X=c_{0}(\mathbb{Z})$, then $F_{w}: X \rightarrow X\left(B_{w}: X \rightarrow X\right)$ denotes the bilateral weighted forward (backward) shift on $X$ given by

$$
F_{w}\left(\left(x_{n}\right)_{n \in \mathbb{Z}}\right)=\left(w_{n-1} x_{n-1}\right)_{n \in \mathbb{Z}} \quad\left(B_{w}\left(\left(x_{n}\right)_{n \in \mathbb{Z}}\right)=\left(w_{n+1} x_{n+1}\right)_{n \in \mathbb{Z}}\right),
$$

where $w=\left(w_{n}\right)_{n \in \mathbb{Z}}$ is a bounded sequence of scalars, called a weight sequence. Recall that

$$
F_{w}\left(B_{w}\right) \text { is invertible } \Longleftrightarrow \inf _{n \in \mathbb{Z}}\left|w_{n}\right|>0
$$


In the case $X=\ell_{p}(\mathbb{N})(1 \leq p<\infty)$ or $X=c_{0}(\mathbb{N})$, we also denote by $F_{w}: X \rightarrow X\left(B_{w}: X \rightarrow X\right)$ the unilateral weighted forward (backward) shift on $X$ with weight sequence $w=\left(w_{n}\right)_{n \in \mathbb{N}}$, which is defined by

$$
F_{w}\left(\left(x_{1}, x_{2}, \ldots\right)\right)=\left(0, w_{1} x_{1}, w_{2} x_{2}, \ldots\right) \quad\left(B_{w}\left(\left(x_{1}, x_{2}, \ldots\right)\right)=\left(w_{2} x_{2}, w_{3} x_{3}, \ldots\right)\right) .
$$

We remark that in this case the weight sequence $w$ is also assumed to be bounded.

\section{Shadowing for operators}

As mentioned in the Introduction, it is an open problem whether the shadowing property implies hyperbolicity for invertible operators on Banach (or Hilbert) spaces. Our main goal in this section is to give a negative answer to this problem. We will see in the next section that hyperbolic operators do not exhibit chaotic behavior. Indeed, they are not even $\mathrm{Li}-$ Yorke chaotic, which is a very weak notion of chaos. Hence, in order to give a negative answer to the above problem, it would be enough to find a Li-Yorke chaotic operator with the shadowing property. Nevertheless, the example we are going to present is actually much better in the sense that it satisfies a very strong chaotic property, called the frequent hypercyclicity criterion, which simultaneously implies frequent hypercyclicity, Devaney chaos, mixing and dense distributional chaos; see $[18$, Section 9.2], [2] and [7, Corollary 20].

Theorem A. Let $T$ be an invertible operator on a Banach space $X$. Suppose that $X=M \oplus N$, where $M$ and $N$ are closed subspaces of $X$ with $T(M) \subset M$ and $T^{-1}(N) \subset N$. If $\sigma\left(\left.T\right|_{M}\right) \subset \mathbb{D}$ and $\sigma\left(\left.T^{-1}\right|_{N}\right) \subset \mathbb{D}$, then $T$ has the shadowing property, the limit shadowing property and the $\ell_{p}$ shadowing property for all $1 \leq p<\infty$.

As an immediate consequence, we have the following:

Corollary 8. Every invertible hyperbolic operator $T$ on a Banach space $X$ has the shadowing property, the limit shadowing property and the $\ell_{p}$ shadowing property for all $1 \leq p<\infty$.

We recall that it was already known that invertible hyperbolic operators have the shadowing property (see [22], for instance).

We also establish the existence of nonhyperbolic invertible operators with the shadowing property.

Recall that an operator $T$ on a separable Banach space $X$ is said to satisfy the frequent hypercyclicity criterion if there are a dense set $X_{0} \subset X$ and a map $S: X_{0} \rightarrow X_{0}$ such that, for all $x \in X_{0}$, the series $\sum_{n=0}^{\infty} T^{n} x$ and $\sum_{n=0}^{\infty} S^{n} x$ converge unconditionally and $T S x=x$.

Theorem B. Let $X=\ell_{q}(\mathbb{Z})(1 \leq q<\infty)$ or $X=c_{0}(\mathbb{Z})$. There exists an invertible weighted shift $T$ on $X$ which satisfies the frequent hypercyclicity criterion (hence it is not hyperbolic) and has the shadowing property, the limit shadowing property and the $\ell_{p}$ shadowing property for all $1 \leq p<\infty$. Moreover any weighted shift operator sufficiently close to $T$ also satisfies the thesis of previous statement.

The next remarks highlight the differences between nonlinear finite dimensional dynamics and infinite dimensional linear dynamics, explaining the status of the shadowing property for finite dimensional diffeomorphisms and raising a question.

\section{Remark 9.}

(a) As commented in the Introduction, for $C^{1}$ diffeomorphisms on a finite dimensional manifold, Lipschitz shadowing is equivalent to hyperbolicity [25]. Our example proves that this is not the case for infinite dimensional linear dynamics. In some sense, this shows that when one considers infinite dimensional spaces, even linear dynamics is richer than nonlinear finite dimensional dynamics. 
(b) The previous theorem resembles a result in [20] where the existence of a nonhyperbolic yet having the shadowing property $C^{\infty}$ diffeomorphism on a surface is exhibited. In view of the results in [25], the shadowing property cannot be Lipschitz shadowing. Indeed, in examples provided in [20] the shadowing property is only $\alpha$-Hölder for some $\alpha<1$.

(c) It is worth pointing out that finite dimensional diffeomorphisms induce infinite dimensional operators: for any diffeomorphism one obtains finite dimensional linear cocycles provided by the derivative of that diffeomorphism and those linear cocycles can be recast as an infinite dimensional linear map (see [10] for discussions of linear cocycles). In particular, the proof in [25] is based on analyzing the dynamics of a diffeomorphism as a linear cocycle, showing that the Lipschitz shadowing implies shadowing for the cocycle of linear maps and from there concluding hyperbolicity using the results in [26]. On the other hand, the spectrum problem related to certain nonlinear infinite dimensional operators, as the discrete Schrödinger operator, can be reduced to a linear cocycle (see [13] for instance).

(d) It is shown in Theorem B that the shadowing property is satisfied for an open set of weighted shifts. It is natural to wonder if the same holds when one considers open sets of linear operators; in particular, is the shadowing property satisfied for any linear operator close to the ones that satisfy Theorem B?

Before we prove Theorems A and B, we will establish two lemmas. In view of Corollary 7, we will tacitly assume complex scalars in what follows.

Lemma 10. (see [23]) An invertible operator $T$ on a Banach space $X$ has the shadowing property if and only if there is a constant $K>0$ such that for every bounded sequence $\left(z_{n}\right)_{n \in \mathbb{Z}}$ in $X$, there is a sequence $\left(y_{n}\right)_{n \in \mathbb{Z}}$ in $X$ such that

$$
\sup _{n \in \mathbb{Z}}\left\|y_{n}\right\| \leq K \sup _{n \in \mathbb{Z}}\left\|z_{n}\right\| \quad \text { and } \quad y_{n+1}=T y_{n}+z_{n} \quad \text { for all } n \in \mathbb{Z}
$$

Proof. Assume that $T$ has the shadowing property and let $\delta>0$ be the constant that appears in the definition of shadowing associated to $\varepsilon=1$. Consider a bounded sequence $\left(z_{n}\right)_{n \in \mathbb{Z}}$ and put $L=\sup _{n \in \mathbb{Z}}\left\|z_{n}\right\|$. Let $\left(x_{n}\right)_{n \in \mathbb{Z}}$ be such that $x_{n+1}=T x_{n}+\frac{\delta}{L} z_{n}$ for all $n \in \mathbb{Z}$. Observe that $\left(x_{n}\right)_{n \in \mathbb{Z}}$ is completely determined by $x_{0}$. Then $\left(x_{n}\right)_{n \in \mathbb{Z}}$ is a $\delta$-pseudotrajectory of $T$. By hypothesis, there is $x \in X$ such that $\left\|x_{n}-T^{n} x\right\|<1$ for all $n \in \mathbb{Z}$. By putting $y_{n}=\frac{L}{\delta}\left(x_{n}-T^{n} x\right)$, we have that (1) hold (with $K=1 / \delta$ ). For the converse, it is enough to consider $\varepsilon=1$ (Remark 5). Put $\delta=\frac{1}{2 K}$ and let $\left(x_{n}\right)_{n \in \mathbb{Z}}$ be a $\delta$-pseudotrajectory of $T$. Put $z_{n}=x_{n+1}-T x_{n}$. By hypothesis, there exists $\left(y_{n}\right)_{n \in \mathbb{Z}}$ such that (1) hold. Since $x_{n+1}-y_{n+1}=T\left(x_{n}-y_{n}\right)$, it follows that $x_{n}-y_{n}=T^{n}\left(x_{0}-y_{0}\right)$. Thus,

$$
\left\|x_{n}-T^{n}\left(x_{0}-y_{0}\right)\right\|=\left\|y_{n}\right\| \leq K \sup _{j \in \mathbb{Z}}\left\|z_{j}\right\| \leq K \delta=1,
$$

for all $n \in \mathbb{Z}$.

Lemma 11. An invertible operator $T$ on a Banach space $X$ has the limit shadowing property (the $\ell_{p}$ shadowing property) if and only if for every sequence $\left(z_{n}\right)_{n \in \mathbb{Z}}$ in $X$ with

$$
\lim _{|n| \rightarrow \infty}\left\|z_{n}\right\|=0 \quad\left(\sum_{n \in \mathbb{Z}}\left\|z_{n}\right\|^{p}<\infty\right)
$$

there exists a sequence $\left(y_{n}\right)_{n \in \mathbb{Z}}$ in $X$ such that

$$
\lim _{|n| \rightarrow \infty}\left\|y_{n}\right\|=0 \quad\left(\sum_{n \in \mathbb{Z}}\left\|y_{n}\right\|^{p}<\infty\right)
$$


and

$$
y_{n+1}=T y_{n}+z_{n} \quad \text { for all } n \in \mathbb{Z}
$$

Proof. Assume that $T$ has the limit shadowing property (the $\ell_{p}$ shadowing property) and consider a sequence $\left(z_{n}\right)_{n \in \mathbb{Z}}$ satisfying (2). Let $\left(x_{n}\right)_{n \in \mathbb{Z}}$ be such that $x_{n+1}=T x_{n}+z_{n}$ for all $n \in \mathbb{Z}$. Then, by hypothesis, there exists $x \in X$ such that $\lim _{|n| \rightarrow \infty}\left\|x_{n}-T^{n} x\right\|=0\left(\sum_{n \in \mathbb{Z}}\left\|x_{n}-T^{n} x\right\|^{p}<\infty\right)$. Hence, by putting $y_{n}=x_{n}-T^{n} x$, we have that (3) and (4) hold. For the converse, consider $\left(x_{n}\right)_{n \in \mathbb{Z}}$ such that

$$
\lim _{|n| \rightarrow \infty}\left\|x_{n+1}-T x_{n}\right\|=0 \quad\left(\sum_{n \in \mathbb{Z}}\left\|x_{n+1}-T x_{n}\right\|^{p}<\infty\right) .
$$

Put $z_{n}=x_{n+1}-T x_{n}$. Then $\left(z_{n}\right)_{n \in \mathbb{Z}}$ satisfies (2). Hence, by hypothesis, there is $\left(y_{n}\right)_{n \in \mathbb{Z}}$ such that $(3)$ and (4) hold. Since $x_{n}-T^{n}\left(x_{0}-y_{0}\right)=y_{n}$, we are done.

Proof of Theorem A. For each $x \in X$, take $x^{(1)} \in M$ and $x^{(2)} \in N$ satisfying $x=x^{(1)}+x^{(2)}$ (note that $x^{(1)}, x^{(2)}$ are unique). There is a constant $\beta>0$ such that

$$
\left\|x^{(1)}\right\| \leq \beta\|x\| \text { and }\left\|x^{(2)}\right\| \leq \beta\|x\| \quad \text { for all } x \in X .
$$

By hypothesis, $r\left(\left.T\right|_{M}\right)<1$ and $r\left(\left.T^{-1}\right|_{N}\right)<1$. Choose $t \in \mathbb{R}$ such that

$$
\max \left\{r\left(\left.T\right|_{M}\right), r\left(\left.T^{-1}\right|_{N}\right)\right\}<t<1
$$

It follows from the spectral radius formula that there exists a constant $C \geq 1$ such that

$$
\left\|\left(\left.T\right|_{M}\right)^{n}\right\| \leq C t^{n} \text { and }\left\|\left(\left.T^{-1}\right|_{N}\right)^{n}\right\| \leq C t^{n} \quad \text { for all } n \in \mathbb{N}_{0}
$$

Consider a bounded sequence $\left(z_{n}\right)_{n \in \mathbb{Z}}$ in $X$. For each $n \in \mathbb{Z}$, we define

$$
y_{n}^{(1)}=\sum_{k=0}^{\infty} T^{k} z_{n-k-1}^{(1)} \in M, \quad y_{n}^{(2)}=-\sum_{k=1}^{\infty} T^{-k} z_{n+k-1}^{(2)} \in N
$$

and

$$
y_{n}=y_{n}^{(1)}+y_{n}^{(2)} \in X .
$$

An easy computation shows that second part of (1) (which is the same as (4)) holds. Moreover,

$$
\left\|y_{n}^{(1)}\right\| \leq C \sum_{k=0}^{\infty} t^{k}\left\|z_{n-k-1}^{(1)}\right\| \quad \text { and } \quad\left\|y_{n}^{(2)}\right\| \leq C \sum_{k=1}^{\infty} t^{k}\left\|z_{n+k-1}^{(2)}\right\| .
$$

Hence,

$$
\sup _{n \in \mathbb{Z}}\left\|y_{n}\right\| \leq\left(\frac{2 \beta C}{1-t}\right) \sup _{n \in \mathbb{Z}}\left\|z_{n}\right\|
$$

which proves that $T$ has the shadowing property by Lemma 10 .

Now, assume that $\left(z_{n}\right)_{n \in \mathbb{Z}}$ satisfies (2). By Lemma 11, it remains to show that (3) holds. By (5), for each $j \in \mathbb{N}$ and each $i \in\{1,2\}$, 


$$
\left\|y_{n}^{(i)}\right\| \leq C\left(\sum_{k=0}^{j} t^{k}\right)\left(\sup _{0 \leq k \leq j}\left\|z_{n+(-1)^{i} k-1}^{(i)}\right\|\right)+C\left(\sum_{k=j+1}^{\infty} t^{k}\right)\left(\sup _{k \in \mathbb{Z}}\left\|z_{k}^{(i)}\right\|\right)
$$

which shows that $\lim _{|n| \rightarrow \infty}\left\|y_{n}\right\|=0$ whenever $\lim _{|n| \rightarrow \infty}\left\|z_{n}\right\|=0$. In the case $p=1$, it is enough to use the estimates

$$
\sum_{n \in \mathbb{Z}}\left\|y_{n}^{(i)}\right\| \leq C \sum_{n \in \mathbb{Z}} \sum_{k=0}^{\infty} t^{k}\left\|z_{n+(-1)^{i} k-1}^{(i)}\right\|=C\left(\sum_{k=0}^{\infty} t^{k}\right)\left(\sum_{n \in \mathbb{Z}}\left\|z_{n}^{(i)}\right\|\right) \quad(i \in\{1,2\}) .
$$

Finally, in the case $1<p<\infty$, we consider its conjugate exponent $q$ (i.e., $1 / p+1 / q=1$ ) and apply Hölder's inequality to obtain

$$
\left\|y_{n}^{(i)}\right\| \leq C\left(\sum_{k=0}^{\infty} t^{\frac{q k}{2}}\right)^{\frac{1}{q}}\left(\sum_{k=0}^{\infty} t^{\frac{p k}{2}}\left\|z_{n+(-1)^{i} k-1}^{(i)}\right\|^{p}\right)^{\frac{1}{p}} \quad(i \in\{1,2\}) .
$$

As a consequence,

$$
\sum_{n \in \mathbb{Z}}\left\|y_{n}^{(i)}\right\|^{p} \leq C^{p}\left(\sum_{k=0}^{\infty} t^{\frac{q k}{2}}\right)^{\frac{p}{q}}\left(\sum_{k=0}^{\infty} t^{\frac{p k}{2}}\right)\left(\sum_{n \in \mathbb{Z}}\left\|z_{n}^{(i)}\right\|^{p}\right) \quad(i \in\{1,2\}) .
$$

Thus, in all cases, (3) holds.

Proof of Theorem B. Fix a real number $\alpha>1$ and let $T$ be the bilateral weighted forward shift on $X$ whose weight sequence $\left(w_{n}\right)_{n \in \mathbb{Z}}$ is given by $w_{n}=\alpha$ if $n<0$ and $w_{n}=1 / \alpha$ if $n \geq 0$. By applying Theorem A with

$$
M=\left\{\left(x_{n}\right)_{n \in \mathbb{Z}}: x_{n}=0 \text { for all } n<0\right\} \quad \text { and } \quad N=\left\{\left(x_{n}\right)_{n \in \mathbb{Z}}: x_{n}=0 \text { for all } n \geq 0\right\},
$$

we see that $T$ has all the above-mentioned shadowing properties. Moreover, in order to see that $T$ satisfies the frequent hypercyclicity criterion, it is enough to consider $X_{0}$ as the set of all sequences $\left(x_{n}\right)_{n \in \mathbb{Z}}$ with finite support and $S=T^{-1}$. To conclude the second part of the thesis, observe that any weighted shift close to $T$ also fits in Theorem A (with the same $M$ and $N$ ) and satisfies the frequent hypercyclicity criterion.

In the remark below we note that all notions of shadowing considered here coincide in the finite dimensional setting. As mentioned before the equivalence (i) $\Leftrightarrow$ (ii) was already known (see [22], where further references can be found). The other equivalences are not so difficult exercises (for instance, we have done them on the arXiv version of this paper).

Remark 12. Fix $p \in[1, \infty)$. If $T$ is an invertible operator on a finite dimensional Banach space $X$, then the following assertions are equivalent:

(i) $T$ is hyperbolic;

(ii) $T$ has the shadowing property;

(iii) $T$ has the limit shadowing property;

(iv) $T$ has the $\ell_{p}$ shadowing property.

We now turn our attention to the case of noninvertible maps. If $h$ is a continuous self-map of a metric space $M$, we can define the notion of positive shadowing simply by replacing the set $\mathbb{Z}$ by the set $\mathbb{N}_{0}$ in the definition of shadowing. Similarly, we can define the notions of positive limit shadowing and positive $\ell_{p}$ 
shadowing for such a map $h$. Remark 5, Proposition 6 and Corollary 7 have analogous statements for not necessarily invertible operators if we add the word "positive" to the corresponding notions of shadowing.

Theorem 13. Let $T$ be a (not necessarily invertible) operator on a Banach space $X$. If $T$ is hyperbolic, then $T$ has the positive shadowing property, the positive limit shadowing property and the positive $\ell_{p}$ shadowing property for all $1 \leq p<\infty$.

Proof. We divide the proof into three cases.

Case 1. $\sigma(T) \subset \mathbb{D}$.

There are $t \in(0,1)$ and $C \geq 1$ such that $\left\|T^{n}\right\| \leq C t^{n}$ for all $n \in \mathbb{N}_{0}$. Given $\varepsilon>0$, put $\delta=\frac{(1-t) \varepsilon}{C}$. Let $\left(x_{n}\right)_{n \in \mathbb{N}_{0}}$ be a $\delta$-pseudotrajectory of $T$ and define $y_{n}=x_{n}-T x_{n-1}$. Then

$$
x_{n}=T^{n} x_{0}+T^{n-1} y_{1}+T^{n-2} y_{2}+\cdots+T y_{n-1}+y_{n} \quad \text { for all } n \in \mathbb{N} .
$$

Since $\left\|y_{n}\right\| \leq \delta$ for all $n \in \mathbb{N}$, we conclude that

$$
\left\|x_{n}-T^{n} x_{0}\right\| \leq C t^{n-1} \delta+C t^{n-2} \delta+\cdots+C t \delta+\delta<\frac{C \delta}{1-t}=\varepsilon \quad(n \in \mathbb{N})
$$

Hence, $\left(x_{n}\right)_{n \in \mathbb{N}_{0}}$ is $\varepsilon$-shadowed by $\left(T^{n} x_{0}\right)_{n \in \mathbb{N}_{0}}$ and $T$ has the positive shadowing property.

Let $\left(x_{n}\right)_{n \in \mathbb{N}_{0}}$ be a sequence in $X$ with $\lim _{n \rightarrow \infty}\left\|T x_{n}-x_{n+1}\right\|=0$. Let $y_{n}$ be defined as above. By (6),

$$
\left\|x_{n}-T^{n} x_{0}\right\| \leq C\left(\sum_{k=0}^{j} t^{k}\right)\left(\sup _{0 \leq k \leq j}\left\|y_{n-k}\right\|\right)+C\left(\sum_{k=j+1}^{n-1} t^{k}\right)\left(\sup _{k \in \mathbb{N}}\left\|y_{k}\right\|\right),
$$

whenever $0<j<n$. Since $\left\|y_{n}\right\| \rightarrow 0$, the above estimate implies that $\left\|x_{n}-T^{n} x_{0}\right\| \rightarrow 0$ as well. Thus, $T$ has the positive limit shadowing property.

Now, suppose that $\sum_{n=0}^{\infty}\left\|T x_{n}-x_{n+1}\right\|<\infty$. Then, by (6),

$$
\sum_{n=0}^{\infty}\left\|x_{n}-T^{n} x_{0}\right\| \leq C \sum_{n=1}^{\infty} \sum_{k=0}^{n-1} t^{k}\left\|y_{n-k}\right\|=C\left(\sum_{k=0}^{\infty} t^{k}\right)\left(\sum_{n=1}^{\infty}\left\|y_{n}\right\|\right)<\infty .
$$

Finally, suppose that $1<p<\infty$ and that $\sum_{n=0}^{\infty}\left\|T x_{n}-x_{n+1}\right\|^{p}<\infty$. By (6),

$$
\left\|x_{n}-T^{n} x_{0}\right\| \leq C \sum_{k=0}^{n-1} t^{k}\left\|y_{n-k}\right\| \leq C\left(\sum_{k=0}^{n-1} t^{\frac{q k}{2}}\right)^{\frac{1}{q}}\left(\sum_{k=0}^{n-1} t^{\frac{p k}{2}}\left\|y_{n-k}\right\|^{p}\right)^{\frac{1}{p}}
$$

where $q$ is the conjugate exponent to $p$. Thus,

$$
\sum_{n=0}^{\infty}\left\|x_{n}-T^{n} x_{0}\right\|^{p} \leq C^{p}\left(\sum_{k=0}^{\infty} t^{\frac{q k}{2}}\right)^{\frac{p}{q}}\left(\sum_{k=0}^{\infty} t^{\frac{p k}{2}}\right)\left(\sum_{n=1}^{\infty}\left\|y_{n}\right\|^{p}\right)<\infty .
$$

Therefore, $T$ also has the positive $\ell_{p}$ shadowing property.

Case 2. $\sigma(T) \subset \mathbb{C} \backslash \overline{\mathbb{D}}$.

Then $T$ is invertible and we can apply Corollary 8. 
Case 3. $\sigma(T) \cap \mathbb{D} \neq \emptyset$ and $\sigma(T) \cap(\mathbb{C} \backslash \overline{\mathbb{D}}) \neq \emptyset$.

In this case, the sets $\sigma_{1}=\sigma(T) \cap \mathbb{D}$ and $\sigma_{2}=\sigma(T) \cap(\mathbb{C} \backslash \overline{\mathbb{D}})$ form a partition of $\sigma(T)$ into two nonempty closed sets. By the Riesz decomposition theorem [18, Theorem B.9], there are nontrivial $T$-invariant closed subspaces $M_{1}$ and $M_{2}$ of $X$ such that

$$
X=M_{1} \oplus M_{2}, \quad \sigma\left(\left.T\right|_{M_{1}}\right)=\sigma_{1} \quad \text { and } \quad \sigma\left(\left.T\right|_{M_{2}}\right)=\sigma_{2} .
$$

By Cases 1 and 2, both $\left.T\right|_{M_{1}}$ and $\left.T\right|_{M_{2}}$ have the positive shadowing property, the positive limit shadowing property and the positive $\ell_{p}$ shadowing property for all $1 \leq p<\infty$, from which it follows easily that $T$ also has these properties.

Remark 14. The converse of Theorem 13 is false in general, the operator constructed in the proof of Theorem $\mathrm{B}$ has all the positive shadowing properties, but it is not hyperbolic.

Let us now prove that all notions of positive shadowing considered here coincide with hyperbolicity in the case of compact operators.

Theorem 15. Fix $p \in[1, \infty)$. If $T$ is a compact operator on a Banach space $X$, then the following assertions are equivalent:

(i) T is hyperbolic;

(ii) $T$ has the positive shadowing property;

(iii) $T$ has the positive limit shadowing property;

(iv) $T$ has the positive $\ell_{p}$ shadowing property.

Proof. Suppose that $T$ has the positive shadowing property (the positive limit shadowing property, the positive $\ell_{p}$ shadowing property). We have to prove that $T$ is hyperbolic. We may assume that $X$ is infinite dimensional (the finite dimensional case is an exercise analogous to that one stated in Remark 12) and that $\sigma(T)$ is not contained in $\mathbb{D}$. Since $T$ is a compact operator, it follows that the sets $\sigma_{1}=\sigma(T) \cap \mathbb{D}$ and $\sigma_{2}=\sigma(T) \backslash \mathbb{D}$ form a partition of $\sigma(T)$ into two nonempty closed sets. By the Riesz decomposition theorem, there are nontrivial $T$-invariant closed subspaces $M_{1}$ and $M_{2}$ of $X$ such that $X=M_{1} \oplus M_{2}, \sigma\left(\left.T\right|_{M_{1}}\right)=\sigma_{1}$ and $\sigma\left(\left.T\right|_{M_{2}}\right)=\sigma_{2}$. The compactness of $T$ also implies that $M_{2}$ is finite dimensional. Hence, since $\left.T\right|_{M_{2}}$ has the positive shadowing property (the positive limit shadowing property, the positive $\ell_{p}$ shadowing property), we have that $\sigma_{2} \cap \mathbb{T}=\emptyset$. Thus, $\sigma(T) \cap \mathbb{T}=\emptyset$.

\section{Expansive operators}

We start this section asserting that uniformly expansive operators do not exhibit chaotic behavior.

Theorem C. A uniformly (positively) expansive operator on a Banach space cannot be Li-Yorke chaotic. In particular, it cannot be hypercyclic.

We also investigate the relationship between expansivity and spectrum.

Theorem D. If $T$ is an operator on a Banach space $X$, then

$$
T \text { uniformly positively expansive } \Rightarrow \sigma_{a}(T) \cap \overline{\mathbb{D}}=\emptyset .
$$

The converse holds if $\rho(T) \cap \mathbb{D} \neq \emptyset$. In particular, the converse holds if $T$ is invertible. 
Before providing the proofs, we state a series of remarks that helps to provide a context to the main theorems of the present section.

Remark 16. The fact that uniformly expansive operators cannot be hypercyclic can be seen by means of a spectral argument. Indeed, if $T$ is hypercyclic, then $\sigma(T) \cap \mathbb{T} \neq \emptyset$ and $\sigma_{p}\left(T^{*}\right)=\emptyset$ (see [3]). Since $\sigma_{r}(T) \subset \sigma_{p}\left(T^{*}\right)$ and $\sigma(T)=\sigma_{r}(T) \cup \sigma_{a}(T)$, we conclude that $\sigma_{a}(T) \cap \mathbb{T} \neq \emptyset$ and thus $T$ is not uniformly expansive.

On the other hand, there exist supercyclic uniformly expansive operators, as we shall see in the remark below. A simple concrete example of such an operator on the Hilbert space $\ell_{2}$ will be given in Example 38 .

Remark 17. Every infinite dimensional separable Banach space admits an invertible operator which is uniformly positively expansive and supercyclic.

Indeed, it is well-known that every infinite dimensional separable Banach space $X$ supports a hypercyclic invertible operator $S$ (see [18, Section 8.2]). Since any nonzero scalar multiple of a supercyclic operator is a supercyclic operator, $T=2\left\|S^{-1}\right\| S$ is a supercyclic operator on $X$. Moreover, since $\left\|T^{-1}\right\|=\frac{1}{2}<1, T$ is a proper dilation. In particular, $T$ is uniformly positively expansive.

Remark 18. A positively expansive operator can be Li-Yorke chaotic. For example, Beauzamy [5] and Prăjitură [27] constructed examples of completely irregular operators on the Hilbert space $\ell_{2}$. These are operators with the property that every nonzero vector is irregular. It follows from Proposition 19(a) and [8, Theorem 34] that such operators are simultaneously positively expansive and generically Li-Yorke chaotic. Also, Read [28] constructed an operator $T$ on $\ell_{1}$ with all nonzero vectors hypercyclic. This operator is simultaneously positively expansive, generically Li-Yorke chaotic and hypercyclic. Moreover, we shall see later an example of an invertible operator on the Hilbert space $\ell_{2}$ which is positively expansive (hence expansive) and hypercyclic (Example 34).

In order to prove Theorems $\mathrm{C}$ and $\mathrm{D}$ we will use the next proposition.

Proposition 19. Let $T$ be an operator on a Banach space $X$. Then:

(a) $T$ is positively expansive $\Leftrightarrow \sup _{n \in \mathbb{N}}\left\|T^{n} x\right\|=\infty$ for every nonzero $x \in X$.

(b) $T$ is uniformly positively expansive $\Leftrightarrow \lim _{n \rightarrow \infty}\left\|T^{n} x\right\|=\infty$ uniformly on $S_{X}$.

If, in addition, $T$ is invertible, then:

(c) $T$ is expansive $\Leftrightarrow \sup _{n \in \mathbb{Z}}\left\|T^{n} x\right\|=\infty$ for every nonzero $x \in X$.

(d) $T$ is uniformly expansive $\Leftrightarrow S_{X}=A \cup B$ where $\lim _{n \rightarrow \infty}\left\|T^{n} x\right\|=\infty$ uniformly on $A$ and $\lim _{n \rightarrow \infty}\left\|T^{-n} x\right\|=\infty$ uniformly on $B$.

Proof. We will only prove item (d). Since the sufficiency of the condition is clear, let us prove its necessity. Assume $T$ uniformly expansive and let $n \in \mathbb{N}$ be as in Definition 2. Let

$$
A=\left\{x \in S_{X}:\left\|T^{n} x\right\| \geq 2\right\} \quad \text { and } \quad B=\left\{x \in S_{X}:\left\|T^{-n} x\right\| \geq 2\right\} .
$$

Then, $S_{X}=A \cup B$. We claim that $\frac{T^{n} x}{\left\|T^{n} x\right\|} \in A$ whenever $x \in A$. Indeed, if $x \in A$ and $y=\frac{T^{n} x}{\left\|T^{n} x\right\|} \notin A$, then $y \in B$ and so $\left\|T^{-n} y\right\| \geq 2$, implying that $\|x\| \geq 2\left\|T^{n} x\right\| \geq 4$, a contradiction. Hence, given $x \in A$, we can define inductively a sequence $\left(x_{k}\right)_{k \in \mathbb{N}}$ in $A$ by putting $x_{1}=x$ and $x_{k}=\frac{T^{n} x_{k-1}}{\left\|T^{n} x_{k-1}\right\|}$ for $k \geq 2$. Clearly, 


$$
x_{k}=\frac{T^{(k-1) n} x}{\left\|T^{n} x_{1}\right\| \cdot \ldots \cdot\left\|T^{n} x_{k-1}\right\|} \quad \text { for all } k \in \mathbb{N} \text {. }
$$

Since $\left\|T^{n} x_{k}\right\| \geq 2$, we obtain $\left\|T^{k n} x\right\| \geq 2\left\|T^{n} x_{1}\right\| \cdot \ldots \cdot\left\|T^{n} x_{k-1}\right\| \geq 2^{k}$ for all $k \in \mathbb{N}$. Let $C=$ $\max _{0 \leq j \leq n-1}\left\|T^{j}\right\| \geq 1$. For each $m \in \mathbb{N}$, we can write $m=k_{m} n-j_{m}$ with unique $k_{m} \in \mathbb{N}$ and $j_{m} \in\{0, \ldots, n-1\}$, and so $\left\|T^{m} x\right\| \geq \frac{2^{k_{m}}}{\left\|T^{j_{m}}\right\|} \geq \frac{2^{k_{m}}}{C}$. Since $x \in A$ is arbitrary and $k_{m} \rightarrow \infty$ as $m \rightarrow \infty$, we conclude that $\lim _{m \rightarrow \infty}\left\|T^{m} x\right\|=\infty$ uniformly on $A$. The proof that $\lim _{m \rightarrow \infty}\left\|T^{-m} x\right\|=\infty$ uniformly on $B$ is analogous.

Remark 20. The sets $A$ and $B$ in Proposition 19(d) can be chosen to be disjoint or to be both closed in $S_{X}$ or to be both open in $S_{X}$.

As we saw in Remark 17, a uniformly expansive operator can be supercyclic. However, this is not the case for hyperbolic operators with nontrivial hyperbolic splittings.

Proposition 21. If $T$ is a hyperbolic operator with nontrivial hyperbolic splitting, then $T$ is not supercyclic.

Proof. By hypothesis, the hyperbolic splitting $X=X_{s} \oplus X_{u}, T=T_{s} \oplus T_{u}$, satisfies $X_{s} \neq\{0\}$ and $X_{u} \neq\{0\}$. By renorming $X$ we may assume that $\left\|T_{s}\right\|<1$ and $\left\|T_{u}^{-1}\right\|<1$. Each vector $x \in X$ has a unique decomposition $x=x_{s}+x_{u}$ with $x_{s} \in X_{s}$ and $x_{u} \in X_{u}$. Moreover, by the open mapping theorem, the mapping $x \in X \mapsto\left(x_{s}, x_{u}\right) \in X_{s} \times X_{u}$ is an isomorphism. Suppose that $T$ admits a supercyclic vector $y \in X$. It must be true that $y_{s} \neq 0$ and $y_{u} \neq 0$. Since $\left\|T^{n} y_{s}\right\| \rightarrow 0$ and $\left\|T^{n} y_{u}\right\| \rightarrow \infty$, there exists $n_{0} \in \mathbb{N}$ such that $\left\|T^{n} y_{u}\right\| \geq 2\left\|T^{n} y_{s}\right\|$ whenever $n \geq n_{0}$. On one hand, the set $D=\left\{\lambda T^{n} y: \lambda\right.$ is a scalar and $\left.n \geq n_{0}\right\}$ is dense in $X$. But on the other hand, each element $z=\lambda T^{n} y \in D$ has decomposition $z=z_{s}+z_{u}=\lambda T^{n} y_{s}+\lambda T^{n} y_{u}$ satisfying $\left\|z_{u}\right\| \geq 2\left\|z_{s}\right\|$, and so $D$ cannot be dense in $X$. This contradiction proves the proposition.

Remark 22. Another way to see the last result in the case of complex scalars comes from the fact that if $T$ is supercyclic, then there exists $R \geq 0$ such that each connected component of $\sigma(T)$ intersects the (possibly degenerate) circle $\{z \in \mathbb{C}:|z|=R\}$ (see [3]). This is impossible if $T$ is a hyperbolic operator with nontrivial hyperbolic splitting, since the unit circle separates at least two connected components of $\sigma(T)$.

Proof of Theorem C. Let us consider the case of a uniformly expansive (necessarily invertible) operator $T$ on a Banach space $X$ (the case of a uniformly positively expansive (not necessarily invertible) operator is simpler). Write $S_{X}=A \cup B$ as in Proposition 19(d). It was proved in [6, Theorem 5] that $T$ is Li-Yorke chaotic if and only if $T$ admits an irregular vector, that is, a vector $x \in X$ such that $\inf _{n \in \mathbb{N}}\left\|T^{n} x\right\|=0$ and $\sup _{n \in \mathbb{N}}\left\|T^{n} x\right\|=\infty$ (hence every hypercyclic operator is Li-Yorke chaotic). Suppose that $T$ is Li-Yorke chaotic and let $y \in S_{X}$ be an irregular vector for $T$. We must have $\frac{T^{k} y}{\left\|T^{k} y\right\|} \in B$ for all $k \in \mathbb{N}$. Indeed, if $\frac{T^{k} y}{\left\|T^{k} y\right\|} \in A$ for some $k \in \mathbb{N}$, then $\lim _{n \rightarrow \infty}\left\|T^{n}\left(\frac{T^{k} y}{\left\|T^{k} y\right\|}\right)\right\|=\infty$, which implies that $\lim _{n \rightarrow \infty}\left\|T^{n} y\right\|=\infty$ and contradicts the fact that $y$ is an irregular vector for $T$. Since $\lim _{n \rightarrow \infty}\left\|T^{-n} x\right\|=\infty$ uniformly on $B$, there exists $n_{0} \in \mathbb{N}$ such that

$$
\left\|T^{-n} x\right\| \geq 2 \quad \text { whenever } x \in B \text { and } n \geq n_{0} .
$$

Since $y$ is an irregular vector for $T$, we can choose $k_{0} \geq n_{0}$ such that $\left\|T^{k_{0}} y\right\| \geq 1$. Now, by choosing $n=k_{0} \geq n_{0}$ and $x=\frac{T^{k_{0}} y}{\left\|T^{k_{0}} y\right\|} \in B$ in (7), we obtain $\|y\| \geq 2\left\|T^{k_{0}} y\right\| \geq 2$. This contradiction proves the theorem.

Proof of Theorem D. It is enough to consider the case of complex scalars. Suppose that there is a point $\lambda \in \sigma_{a}(T) \cap \overline{\mathbb{D}}$. Let $\left(x_{k}\right)_{k \in \mathbb{N}}$ be a sequence in $S_{X}$ such that $\lim _{k \rightarrow \infty}\left\|\lambda x_{k}-T x_{k}\right\|=0$. Since 


$$
\left\|\lambda^{n} x_{k}-T^{n} x_{k}\right\| \leq|\lambda|\left\|\lambda^{n-1} x_{k}-T^{n-1} x_{k}\right\|+\left\|T^{n-1}\right\|\left\|\lambda x_{k}-T x_{k}\right\|
$$

it follows by induction that

$$
\lim _{k \rightarrow \infty}\left\|\lambda^{n} x_{k}-T^{n} x_{k}\right\|=0 \quad \text { for all } n \in \mathbb{N} .
$$

Since $\left\|\lambda^{n} x_{k}\right\| \leq 1$ for all $k, n \in \mathbb{N}$, we conclude from Proposition 19(b) that $T$ is not uniformly positively expansive.

Now, assume that $\rho(T) \cap \mathbb{D} \neq \emptyset$ and $\sigma_{a}(T) \cap \overline{\mathbb{D}}=\emptyset$. Since $\sigma_{a}(T)$ contains the boundary of $\sigma(T)$, we must have $\sigma(T) \cap \overline{\mathbb{D}}=\emptyset$. Hence,

$$
\sigma\left(T^{-1}\right)=\left\{\lambda^{-1}: \lambda \in \sigma(T)\right\} \subset \mathbb{D}
$$

that is, $r\left(T^{-1}\right)<1$. Choose $R \in \mathbb{R}$ such that $r\left(T^{-1}\right)<R<1$. It follows from the spectral radius formula that there exists $n_{0} \in \mathbb{N}$ such that

$$
\left\|T^{n} x\right\| \geq R^{-n}\|x\| \quad \text { for all } x \in X \text { and } n \geq n_{0},
$$

which implies that $T$ is uniformly positively expansive.

Mazur's results revisited: Let us now give short direct proofs of the results obtained in [21]. One of them characterizes normal expansive operators in terms of their spectral properties (Theorem 23) and the other one shows that for invertible normal operators, shadowing is equivalent to hyperbolicity (Corollary 28). We finish the present subsection extending the last result to not necessarily invertible normal operators.

Theorem 23. If $T$ is an invertible normal operator on a Hilbert space $H$, then $T$ is expansive if and only if $\sigma_{p}\left(T^{*} T\right) \cap \mathbb{T}=\emptyset$.

Proof. We may assume complex scalars. Assume $\sigma_{p}\left(T^{*} T\right) \cap \mathbb{T} \neq \emptyset$ and let $\lambda$ be a point in this intersection. There exists $x \in H \backslash\{0\}$ such that $T^{*} T x=\lambda x$. Hence, for every $n \in \mathbb{Z},\left\|T^{n} x\right\|^{2}=\left\langle\left(T^{*} T\right)^{n} x, x\right\rangle=\lambda^{n}\|x\|^{2}$, implying that $\left\|T^{n} x\right\|=\|x\|$. Thus, $T$ is not expansive.

Conversely, suppose that $T$ is not expansive and consider the positive operator $S=T^{*} T$. There exists $x \in S_{H}$ with $\left\|T^{n} x\right\|<2$ for all $n \in \mathbb{Z}$. Since $T$ is normal,

$$
\left\|S^{n} x\right\|=\left\|\left(T^{n}\right)^{*} T^{n} x\right\|=\left\|T^{2 n} x\right\|<2 \quad \text { for all } n \in \mathbb{Z} .
$$

Let $\mu$ be the spectral measure associated to $S$ and $x$. Since $S$ is an invertible positive operator, $\sigma(S) \subset(0, \infty)$. Thus, by the Cauchy-Schwartz inequality,

$$
0 \leq \int_{\sigma(S)} t^{n} d \mu(t)=\left\langle S^{n} x, x\right\rangle \leq\left\|S^{n} x\right\|\|x\|<2 \quad \text { for all } n \in \mathbb{Z} .
$$

For each $\alpha<1$ and each $\beta>1$, let $A_{\alpha}=\sigma(S) \cap(0, \alpha]$ and $B_{\beta}=\sigma(S) \cap[\beta, \infty)$. Since

$$
\alpha^{-n} \mu\left(A_{\alpha}\right) \leq \int_{\sigma(S)} t^{-n} d \mu(t)<2 \quad \text { and } \quad \beta^{n} \mu\left(B_{\beta}\right) \leq \int_{\sigma(S)} t^{n} d \mu(t)<2 \text {, }
$$

for all $n \in \mathbb{N}$, we conclude that $\mu\left(A_{\alpha}\right)=\mu\left(B_{\beta}\right)=0$. This implies that $\sigma(S) \backslash\{1\}$ has $\mu$-measure zero. Therefore, 


$$
\|S x-x\|^{2}=\left\langle(S-I)^{2} x, x\right\rangle=\int_{\sigma(S)}(t-1)^{2} d \mu(t)=0,
$$

and so $1 \in \sigma_{p}(S)$.

Remark 24. Recall that a Hilbert space operator $T$ is said to be hyponormal if

$$
\left\|T^{*} x\right\| \leq\|T x\| \text { for all } x .
$$

It is natural to ask if the previous theorem can be generalized to hyponormal operators. We shall prove in the next section that the implication

$$
\sigma_{p}\left(T^{*} T\right) \cap \mathbb{T}=\emptyset \quad \Longrightarrow \quad T \text { expansive }
$$

holds for hyponormal weighted shifts (Proposition 40), but it is not true in general, and that the converse may fail even for hyponormal weighted shifts (Remark 41).

Remark 25. Let $T$ be a normal operator on a complex Hilbert space $H$. In view of the previous theorem, it is natural to make the following question: Is it true that $T$ is positively expansive if and only if $\sigma_{p}\left(T^{*} T\right) \cap \overline{\mathbb{D}}=\emptyset$ ?

The direct implication is true, since $T^{*} T x=\lambda x$, with $\lambda \in \overline{\mathbb{D}}$ and $x \neq 0$, implies that $\left\|T^{n} x\right\|^{2}=$ $\left\langle\left(T^{*} T\right)^{n} x, x\right\rangle=\lambda^{n}\|x\|^{2} \leq\|x\|^{2}(n \in \mathbb{N})$, and so $T$ is not positively expansive.

However, the converse is not true in general, even if $T$ is invertible. Indeed, let $T: L^{2}[0,1] \rightarrow L^{2}[0,1]$ be defined by

$$
(T f)(t)=\frac{t+1}{2} f(t) \quad \text { for all } f \in L^{2}[0,1]
$$

Then $T$ is invertible, self-adjoint, not positively expansive, and $\sigma_{p}\left(T^{*} T\right)=\emptyset$.

Nevertheless, we have the following characterization.

Proposition 26. Let $T$ be a normal operator on a complex Hilbert space $H$. Then, $T$ is positively expansive if and only if $\mu\left(\sigma\left(T^{*} T\right) \cap(1, \infty)\right)>0$ for every spectral measure $\mu$ associated to $T^{*} T$.

Proof. Let $S=T^{*} T$. If $T$ is not positively expansive, there is $x \in S_{H}$ such that $\left\|T^{n} x\right\|<2$ for all $n \in \mathbb{N}$. By letting $\mu$ be the spectral measure associated to $S$ and $x$, we obtain

$$
0 \leq \int_{\sigma(S)} t^{n} d \mu(t)=\left\langle S^{n} x, x\right\rangle<2 \text { for all } n \in \mathbb{N},
$$

which implies that $\mu(\sigma(S) \cap(1, \infty))=0$. Conversely, suppose that for some $x \neq 0$, the spectral measure $\mu$ associated to $S$ and $x$ satisfies $\mu(\sigma(S) \cap(1, \infty))=0$. Then,

$$
\left\|T^{2 n} x\right\|^{2}=\left\|S^{n} x\right\|^{2}=\left\langle S^{2 n} x, x\right\rangle=\int_{\sigma(S)} t^{2 n} d \mu(t) \leq\|x\|^{2} \quad \text { for all } n \in \mathbb{N},
$$

implying that $T$ is not positively expansive.

We say that a sequence $\left(t_{n}\right)_{n \in \mathbb{Z}}$ of scalars is $O(|n|)$ if there exist $\alpha>0, \beta>0$ and $n_{0} \in \mathbb{N}$ such that $\alpha|n| \leq\left|t_{n}\right| \leq \beta|n|$ whenever $|n| \geq n_{0}$. 
Let us now establish a result which will imply a much simpler and shorter proof of the main result in [21] (see Corollary 28).

Theorem 27. Let $T$ be an invertible operator on a Banach space $X$ such that for all $z \in X$, the sequence $\left(\left\|T^{n} z\right\|\right)_{n \in \mathbb{Z}}$ is not $O(|n|)$. If $T$ has the shadowing property, then $T$ is uniformly expansive.

Proof. Suppose that $T$ has the shadowing property and let $\delta>0$ be the constant that appears in the definition of shadowing associated to $\varepsilon=1$. Assume that $T$ is not uniformly expansive. Then, by [19, Theorem 1], the intersection $\sigma_{a}(T) \cap \mathbb{T}$ is nonempty. Take a scalar $\lambda$ in this intersection. Hence, there exists $x_{0} \in S_{X}$ such that $\left\|\lambda x_{0}-T x_{0}\right\|<\delta / 2$. For each $n \in \mathbb{Z}$, let $y_{n}=2 \lambda^{n} x_{0}$. Then $\left(y_{n}\right)_{n \in \mathbb{Z}}$ is a $\delta$-pseudotrajectory of $T$, and so there exists $y \in X$ such that $\left\|y_{n}-T^{n} y\right\|<1$ for all $n \in \mathbb{Z}$. Therefore, $1<\left\|T^{n} y\right\|<3$ for all $n \in \mathbb{Z}$. Now, let $\left(z_{n}\right)_{n \in \mathbb{Z}}$ be defined by $z_{n}=\frac{n \delta}{3} T^{n} y$. Since $\left(z_{n}\right)_{n \in \mathbb{Z}}$ is a $\delta$-pseudotrajectory of $T$, there exists $z \in X$ such that $\left\|z_{n}-T^{n} z\right\|<1$ for all $n \in \mathbb{Z}$. Thus, $\frac{|n| \delta}{3}-1<\left\|T^{n} z\right\|<|n| \delta+1$ for all $n \in \mathbb{Z}$. This contradicts the fact that $\left(\left\|T^{n} z\right\|\right)_{n \in \mathbb{Z}}$ is not $O(|n|)$.

Corollary 28. If $T$ is an invertible normal operator on a Hilbert space $H$, then $T$ has the shadowing property if and only if $T$ is hyperbolic.

Proof. Suppose that $T$ has the shadowing property but is not hyperbolic. Since $T$ is normal, $\sigma(T)=\sigma_{a}(T)$, and so $T$ is not uniformly expansive. Hence, by Theorem 27 , there exists $z \in H$ such that $\left(\left\|T^{n} z\right\|\right)_{n \in \mathbb{Z}}$ is $O(|n|)$. Let $\alpha>0, \beta>0$ and $n_{0} \in \mathbb{N}$ be such that

$$
\alpha|n| \leq\left\|T^{n} z\right\| \leq \beta|n| \quad \text { whenever }|n| \geq n_{0} .
$$

Consider the invertible positive operator $S=T^{*} T$ and let $\mu$ be the spectral measure associated to $S$ and $z$. Then,

$$
0 \leq \int_{\sigma(S)} t^{n} d \mu(t)=\left\langle S^{n} z, z\right\rangle=\left\|T^{n} z\right\|^{2} \leq \beta^{2}|n|^{2} \quad \text { whenever }|n| \geq n_{0}
$$

By arguing as in the proof of Theorem 23 (with the sets $A_{\alpha}$ and $B_{\beta}$ ), we see that $\sigma(S) \backslash\{1\}$ has $\mu$-measure zero and so $S z=z$. This implies that $\left\|T^{n} z\right\|=\|z\|$ for all $n \in \mathbb{Z}$, which contradicts the first inequality in (8).

Remark 29. The thesis of Theorem 27 can be also obtained replacing the hypothesis about the norm growth on the sequences of iterates by expansiveness. In fact, if $T$ is not uniformly expansive, arguing as in the proof of Theorem 27, one obtains a vector $y \in X$ such that $1<\left\|T^{n} y\right\|<3$ for all $n \in \mathbb{Z}$, contradicting the hypothesis that $T$ is expansive.

Theorem 30. If $T$ is a (not necessarily invertible) normal operator on a Hilbert space $H$, then $T$ has the positive shadowing property if and only if $T$ is hyperbolic.

Proof. Suppose that $T$ has the positive shadowing property. Assume that $T$ is not hyperbolic and argue as in the proof of Theorem 27 to obtain a vector $z \in H$ such that

$$
\frac{n \delta}{3}-1<\left\|T^{n} z\right\|<n \delta+1 \quad \text { for all } n \in \mathbb{N}_{0}
$$

Let $S=T^{*} T$ and let $\mu$ be the spectral measure associated to $S$ and $z$. Since 


$$
0 \leq \int_{\sigma(S)} t^{n} d \mu(t)=\left\langle S^{n} z, z\right\rangle=\left\|T^{n} z\right\|^{2} \leq(n \delta+1)^{2} \quad \text { for all } n \in \mathbb{N}_{0}
$$

it follows that $\sigma(S) \cap(1, \infty)$ has $\mu$-measure zero. Hence, $\left\|T^{n} z\right\| \leq(\mu(\sigma(S)))^{\frac{1}{2}}$ for all $n \in \mathbb{N}_{0}$, which contra$\operatorname{dicts}(9)$.

\section{Expansive weighted shifts}

In this section we obtain complete characterizations of the notions of expansivity for weighted shifts by looking at their weights.

Theorem E. Let $X=\ell_{p}(\mathbb{Z})(1 \leq p<\infty)$ or $X=c_{0}(\mathbb{Z})$, and consider a weight sequence $w=\left(w_{n}\right)_{n \in \mathbb{Z}}$ with $\inf _{n \in \mathbb{Z}}\left|w_{n}\right|>0$.

(1) The following assertions are equivalent:

(i) $F_{w}: X \rightarrow X$ is expansive;

(ii) (a) $F_{w}: X \rightarrow X$ or

(b) $F_{w}^{-1}: X \rightarrow X$ is positively expansive;

(iii) (a) $\sup _{n \in \mathbb{N}}\left|w_{1} \cdot \ldots \cdot w_{n}\right|=\infty$ or

(b) $\sup _{n \in \mathbb{N}}\left|w_{-n} \cdot \ldots \cdot w_{-1}\right|^{-1}=\infty$.

(2) The following assertions are equivalent:

(i) $F_{w}: X \rightarrow X$ is uniformly expansive;

(ii) One of the following conditions holds:

(a) $\lim _{n \rightarrow \infty}\left(\inf _{k \in \mathbb{Z}}\left|w_{k} \cdot \ldots \cdot w_{k+n-1}\right|\right)=\infty$,

(b) $\lim _{n \rightarrow \infty}\left(\inf _{k \in \mathbb{Z}}\left|w_{k-n} \cdot \ldots \cdot w_{k-1}\right|^{-1}\right)=\infty$,

(c) $\lim _{n \rightarrow \infty}\left(\inf _{k \in \mathbb{N}}\left|w_{k} \cdot \ldots \cdot w_{k+n-1}\right|\right)=\infty$ and $\lim _{n \rightarrow \infty}\left(\inf _{k \in-\mathbb{N}}\left|w_{k-n} \cdot \ldots \cdot w_{k-1}\right|^{-1}\right)=\infty$.

Before proving the above theorem we state a lemma.

Lemma 31. Given a nontrivial partition $\{I, J\}$ of $\mathbb{Z}$ (that is, $I \cup J=\mathbb{Z}, I \cap J=\emptyset, I \neq \emptyset$ and $J \neq \emptyset$ ) such that there is a map $\varphi: \mathbb{Z} \rightarrow[0, \infty)$ satisfying

$$
\lim _{n \rightarrow \infty}\left[\inf _{k \in I}(\varphi(k) \cdot \ldots \cdot \varphi(k+n-1))\right]>1 \text { and } \lim _{n \rightarrow \infty}\left[\sup _{k \in J}(\varphi(k-n) \cdot \ldots \cdot \varphi(k-1))\right]<1,
$$

then there exist $i, j \in \mathbb{Z}$ such that $(-\infty, j] \cap \mathbb{Z} \subset J$ and $[i, \infty) \cap \mathbb{Z} \subset I$.

Proof. By hypothesis, there exists $n_{0} \in \mathbb{N}$ such that $\varphi(k) \cdot \ldots \cdot \varphi(k+n-1)>1$ for all $k \in I$, and $\varphi(k-n) \cdot \ldots \cdot \varphi(k-1)<1$ for all $k \in J$, whenever $n \geq n_{0}$. We claim that

$$
k \in I \Rightarrow k+n \in I \text { for all } n \geq n_{0} .
$$

Indeed, suppose that $k \in I$ but $k+n \in J$ for a certain $n \geq n_{0}$. Then,

$$
\varphi(k) \cdot \ldots \cdot \varphi(k+n-1)=\varphi((k+n)-n) \cdot \ldots \cdot \varphi((k+n)-1)
$$

is simultaneously $>1$ and $<1$, because $k \in I, k+n \in J$ and $n \geq n_{0}$. This contradiction proves (10). Analogously, we have that 


$$
k \in J \Rightarrow k-n \in J \text { for all } n \geq n_{0}
$$

Since $I \neq \emptyset$ and $J \neq \emptyset$, it is clear that (10) and (11) imply the existence of $i, j \in \mathbb{Z}$ with the desired properties.

Proof of Theorem E. Let $e_{j}, j \in \mathbb{Z}$, denote the canonical unit vectors in $X$.

(1): If $F_{w}$ is expansive, then Proposition 19(c) implies that

$$
\sup _{n \in \mathbb{N}}\left\|F_{w}^{n}\left(e_{1}\right)\right\|=\infty \quad \text { or } \quad \sup _{n \in \mathbb{N}}\left\|F_{w}^{-n}\left(e_{1}\right)\right\|=\infty .
$$

The first equality means that $\sup _{n \in \mathbb{N}}\left|w_{1} \cdot \ldots \cdot w_{n}\right|=\infty$, whereas the second one means that $\sup _{n \in \mathbb{N}} \mid w_{-n+1}$. $\left.\ldots \cdot w_{0}\right|^{-1}=\infty$, which is clearly equivalent to $\sup _{n \in \mathbb{N}}\left|w_{-n} \cdot \ldots \cdot w_{-1}\right|^{-1}=\infty$. This shows that (i) implies (iii). Now, assume that $\sup _{n \in \mathbb{N}}\left|w_{1} \cdot \ldots \cdot w_{n}\right|=\infty$. Let $x=\left(x_{j}\right)_{j \in \mathbb{Z}}$ be any nonzero vector in $X$ and choose $k \in \mathbb{Z}$ such that $x_{k} \neq 0$. Then,

$$
\sup _{n \in \mathbb{N}}\left\|F_{w}^{n}(x)\right\| \geq \frac{\left|x_{k}\right| \prod_{j=k}^{0}\left|w_{j}\right|}{\prod_{j=1}^{k-1}\left|w_{j}\right|} \sup _{n \in \mathbb{N}}\left|w_{1} \cdot \ldots \cdot w_{k+n-1}\right|=\infty,
$$

where a product over an empty set of indices has value 1 , by definition. Hence, by Proposition 19(a), $F_{w}$ is positively expansive. Analogously, $\sup _{n \in \mathbb{N}}\left|w_{-n} \cdot \ldots \cdot w_{-1}\right|^{-1}=\infty$ implies that $F_{w}^{-1}$ is positively expansive. Thus, (iii) implies (ii). Finally, it is trivial that (ii) implies (i).

(2): Suppose that $F_{w}$ is uniformly expansive. By Proposition $19(\mathrm{~d})$, there is a partition $\{A, B\}$ of $S_{X}$ such that $\lim _{n \rightarrow \infty} c_{n}=\infty$ and $\lim _{n \rightarrow \infty} d_{n}=\infty$, where

$$
c_{n}=\inf _{x \in A}\left\|F_{w}^{n}(x)\right\| \quad \text { and } \quad d_{n}=\inf _{x \in B}\left\|F_{w}^{-n}(x)\right\| \quad(n \in \mathbb{N}) .
$$

We remark that an infimum over an empty set of indices has value $\infty$, by definition. Let

$$
I=\left\{k \in \mathbb{Z}: e_{k} \in A\right\} \quad \text { and } \quad J=\left\{k \in \mathbb{Z}: e_{k} \in B\right\} .
$$

Then $\{I, J\}$ is a partition of $\mathbb{Z}$. Since, for all $n \in \mathbb{N}$,

$$
\inf _{k \in I}\left|w_{k} \cdot \ldots \cdot w_{k+n-1}\right| \geq c_{n} \quad \text { and } \quad \inf _{k \in J}\left|w_{k-n} \cdot \ldots \cdot w_{k-1}\right|^{-1} \geq d_{n}
$$

we conclude that

$$
\lim _{n \rightarrow \infty}\left(\inf _{k \in I}\left|w_{k} \cdot \ldots \cdot w_{k+n-1}\right|\right)=\infty \quad \text { and } \quad \lim _{n \rightarrow \infty}\left(\inf _{k \in J}\left|w_{k-n} \cdot \ldots \cdot w_{k-1}\right|^{-1}\right)=\infty
$$

Thus, $J=\emptyset$ gives the first possibility in (ii) while $I=\emptyset$ gives the second one. Assume that $I \neq \emptyset$ and $J \neq \emptyset$. By Lemma 31, there exist $i, j \in \mathbb{Z}$ such that

$$
(-\infty, j] \cap \mathbb{Z} \subset J \quad \text { and } \quad[i, \infty) \cap \mathbb{Z} \subset I .
$$

Since $w_{k} \neq 0$ for all $k \in \mathbb{Z},(12)$ and (13) imply the third possibility in (ii).

Conversely, assume that (ii) holds. Let $I=\mathbb{Z}$ and $J=\emptyset$, or $I=\emptyset$ and $J=\mathbb{Z}$, or $I=\mathbb{N}$ and $J=-\mathbb{N}_{0}$, depending on whether the first, the second, or the third possibility in (ii) holds, respectively. Then, in any case, (12) holds. Let $n \in \mathbb{N}$ be such that

$$
\inf _{k \in I}\left|w_{k} \cdot \ldots \cdot w_{k+n-1}\right| \geq 4 \text { and } \inf _{k \in J}\left|w_{k-n} \cdot \ldots \cdot w_{k-1}\right|^{-1} \geq 4
$$


Given $x=\left(x_{k}\right)_{k \in \mathbb{Z}} \in S_{X}$, we can write $x=a+b$ where $a=\left(a_{k}\right)_{k \in \mathbb{Z}}$ and $b=\left(b_{k}\right)_{k \in \mathbb{Z}}$ satisfy $a_{k}=0$ whenever $k \in J$ and $b_{k}=0$ whenever $k \in I$. Since $1=\|x\| \leq\|a\|+\|b\|$, we have that $\|a\| \geq \frac{1}{2}$ or $\|b\| \geq \frac{1}{2}$. If $\|a\| \geq \frac{1}{2}$ then

$$
\left\|F_{w}^{n}(x)\right\| \geq\left\|F_{w}^{n}(a)\right\|=\left\|\left(\left(w_{k} \cdot \ldots \cdot w_{k+n-1}\right) a_{k}\right)_{k \in \mathbb{Z}}\right\| \geq 4\|a\| \geq 2
$$

and if $\|b\| \geq \frac{1}{2}$ then

$$
\left\|F_{w}^{-n}(x)\right\| \geq\left\|F_{w}^{-n}(b)\right\|=\left\|\left(\left(w_{k-n} \cdot \ldots \cdot w_{k-1}\right)^{-1} b_{k}\right)_{k \in \mathbb{Z}}\right\| \geq 4\|b\| \geq 2 .
$$

Hence, by definition, $F_{w}$ is uniformly expansive.

Remark 32. In Theorem E, note that (1.ii.a) is equivalent to (1.iii.a), and (1.ii.b) is equivalent to (1.iii.b). In particular, a similar formulation of the theorem characterizes the positively expansive weighted shifts, even in the case where the underlying space is $\mathbb{N}$. Similarly, uniform positive expansivity is equivalent to (2.ii.a) and it is also equivalent to a similar condition where the limit is replaced by the supremum. However, the possibility (2.ii.c) can indeed happen, as can be seen by choosing $w=\left(\ldots, \frac{1}{2}, \frac{1}{2}, \frac{1}{2}, 2,2,2, \ldots\right)$. This shows that $F_{w}$ can be uniformly expansive without $F_{w}$ or $F_{w}^{-1}$ being uniformly positively expansive, in contrast to what happens in the case of expansivity. It is clear that a unilateral weighted backward shift cannot be positively expansive, but positively expansive bilateral weighted backward shifts are characterized by $\sup _{n \in \mathbb{N}}\left|w_{-n} \cdot \ldots \cdot w_{-1}\right|=\infty$ and $w_{j} \neq 0$ for all $j \geq 0$. In the uniform case, the characterization is given by $n \in \mathbb{N}$ $\sup _{n \in \mathbb{N}}\left(\inf _{k \in \mathbb{Z}}\left|w_{k-n+1} \cdot \ldots \cdot w_{k}\right|\right)=\infty$ and similarly replacing the supremum by the limit.

Remark 33. If $T$ is an invertible operator on a Banach space $X$, it is clear that

$$
T \text { or } T^{-1} \text { positively expansive } \Rightarrow T \text { expansive. }
$$

We saw in Theorem $\mathrm{E}(\mathrm{a})$ that the converse holds for the operators $F_{w}$ on the spaces $\ell_{p}(\mathbb{Z})(1 \leq p<\infty)$ or $c_{0}(\mathbb{Z})$. Of course, the converse is not true in general. For instance, if $T$ is any invertible hyperbolic operator with nontrivial hyperbolic splitting, then $T$ is uniformly expansive, but neither $T$ nor $T^{-1}$ is positively expansive.

Let us now see an example of an invertible operator on the Hilbert space $\ell_{2}(\mathbb{Z})$ which is positively expansive and hypercyclic.

Example 34. Fix a real number $t>1$ and consider the weight sequence $w=\left(w_{n}\right)_{n \in \mathbb{Z}}$ given by $w_{n}=t$ for all $n \geq 0$, and

$$
\left(w_{-1}, w_{-2}, w_{-3}, \ldots\right)=\left(t, \frac{1}{t}, \frac{1}{t}, t, t, t, t, \frac{1}{t}, \ldots, \frac{1}{t}, t, \ldots, t, \ldots\right),
$$

where the successive blocks of $t$ 's and $\frac{1}{t}$ 's have lengths $2^{0}, 2^{1}, 2^{2}, \ldots$ Let

$$
m_{k}=2^{0}+2^{1}+\cdots+2^{2 k-1} \quad \text { and } \quad n_{k}=2^{0}+2^{1}+\cdots+2^{2 k} \quad(k \in \mathbb{N}) .
$$

A simple induction argument shows that $w_{-m_{k}} \cdot \ldots \cdot w_{-1} \leq \frac{1}{t^{k}}$ and $w_{-n_{k}} \cdot \ldots \cdot w_{-1} \geq t^{k}$ for all $k \in \mathbb{N}$. In particular, $\sup _{n \in \mathbb{N}}\left(w_{-n} \cdot \ldots \cdot w_{-1}\right)=\infty$. Hence, the bilateral weighted backward shift $B_{w}: \ell_{2}(\mathbb{Z}) \rightarrow$ $\ell_{2}(\mathbb{Z})$ is positively expansive. Since $\inf _{n \in \mathbb{Z}} w_{n}>0, B_{w}$ is invertible. Hence, $B_{w}$ is also expansive. By $[3$, Corollary 1.39], $B_{w}$ is hypercyclic if and only if, for any $q \in \mathbb{N}$, 


$$
\liminf _{n \rightarrow \infty} \max \left\{\left(w_{1} \cdot \ldots \cdot w_{n+q}\right)^{-1},\left(w_{0} \cdot \ldots \cdot w_{-n+q+1}\right)\right\}=0 .
$$

But this condition follows from the fact that

$$
\max \left\{\left(w_{1} \cdot \ldots \cdot w_{\left(m_{k}+q+1\right)+q}\right)^{-1},\left(w_{0} \cdot \ldots \cdot w_{-\left(m_{k}+q+1\right)+q+1}\right)\right\} \leq \frac{1}{t^{k-1}} \quad \text { for all } k \in \mathbb{N} .
$$

Thus, the operator $B_{w}$ is also hypercyclic.

\section{Remark 35.}

(a) Let $X=\ell_{p}(\mathbb{Z})(1 \leq p<\infty)$ or $X=c_{0}(\mathbb{Z})$, and consider a weight sequence $w=\left(w_{n}\right)_{n \in \mathbb{Z}}$ with $\inf _{n \in \mathbb{Z}}\left|w_{n}\right|>0$. It is known (see [11]) that the spectrum of the invertible bilateral weighted forward shift $F_{w}: X \rightarrow X$ is the annulus $\left\{\lambda \in \mathbb{C}: \frac{1}{r\left(F_{w}^{-1}\right)} \leq|\lambda| \leq r\left(F_{w}\right)\right\}$. Since $\left\|F_{w}^{n}\right\|=\sup _{k \in \mathbb{Z}}\left|w_{k} \cdot \ldots \cdot w_{k+n-1}\right|$ and $\left\|F_{w}^{-n}\right\|=\sup _{k \in \mathbb{Z}}\left|w_{k} \cdot \ldots \cdot w_{k+n-1}\right|^{-1}$, we deduce that the following assertions are equivalent:

(i) $F_{w}$ is hyperbolic;

(ii) $\sigma\left(F_{w}\right) \subset \mathbb{D}$ or $\sigma\left(F_{w}^{-1}\right) \subset \mathbb{D}$;

(iii) $\lim _{n \rightarrow \infty} \sup _{k \in \mathbb{Z}}\left|w_{k} \cdot \ldots \cdot w_{k+n-1}\right|^{\frac{1}{n}}<1$ or $\lim _{n \rightarrow \infty} \sup _{k \in \mathbb{Z}}\left|w_{k} \cdot \ldots \cdot w_{k+n-1}\right|^{-\frac{1}{n}}<1$.

(b) Let $A=\mathbb{N}$ or $A=\mathbb{Z}$, let $X=\ell_{p}(A)(1 \leq p<\infty)$ or $X=c_{0}(A)$, and consider a weight sequence $w=\left(w_{n}\right)_{n \in A}$. Let $T$ be either the weighted forward shift $F_{w}: X \rightarrow X$ or the weighted backward shift $B_{w}: X \rightarrow X$. Assume that $T$ is not invertible (this is automatically the case if $A=\mathbb{N}$ ). Since $\sigma(T)$ is equal to the disc $\{\lambda \in \mathbb{C}:|\lambda| \leq r(T)\}$ (see [11]), we deduce that the following assertions are equivalent:

(i) $T$ is hyperbolic;

(ii) $\sigma(T) \subset \mathbb{D}$;

(iii) $\lim _{n \rightarrow \infty} \sup _{k \in A}\left|w_{k} \cdot \ldots \cdot w_{k+n-1}\right|^{\frac{1}{n}}<1$.

Remark 36. The study of expansiveness for invertible bilateral weighted backward shifts can be reduced to the corresponding case of forward shifts (see Theorem E and Remark 35(a)).

\section{Remark 37.}

(a) As mentioned before, it was proved in [16] that every invertible hyperbolic operator is uniformly expansive. Examples of uniformly expansive nonhyperbolic operators were also obtained in [16]. We observe that such examples can be easily obtained by using the characterizations given in Theorem $\mathrm{E}(2)$ and Remark 35(a).

(b) In the case of noninvertible operators, we observe that there is no relation between hyperbolicity and uniform positive expansivity in general. For instance, it follows from Remarks 32 and 35(b) that in the class of unilateral weighted forward shifts on $\ell_{p}(\mathbb{N})(1 \leq p<\infty)$ or on $c_{0}(\mathbb{N})$, the set of hyperbolic shifts is disjoint from the set of positively expansive shifts.

Let us now see a concrete example of an invertible operator on the Hilbert space $\ell_{2}(\mathbb{Z})$ which is uniformly positively expansive and supercyclic.

Example 38. Fix real numbers $\alpha>\beta>1$ and consider the weight sequence

$$
w=\left(w_{n}\right)_{n \in \mathbb{Z}}=(\ldots, \beta, \beta, \beta, \alpha, \alpha, \alpha, \ldots),
$$

where the first $\alpha$ appears at position 1 . Consider the bilateral weighted backward shift $B_{w}: \ell_{2}(\mathbb{Z}) \rightarrow \ell_{2}(\mathbb{Z})$. Since $\left\|B_{w}(x)\right\| \geq \beta\|x\|$ for all $x \in \ell_{2}(\mathbb{Z}), B_{w}$ is uniformly positively expansive. Since $B_{w}$ is invertible, 
$B_{w}$ is also uniformly expansive. By [3, Corollary 1], $B_{w}$ is supercyclic if and only if, for any $q \in \mathbb{N}$, $\liminf _{n \rightarrow \infty} \frac{w_{0} \cdot \ldots \cdot w_{-n+q+1}}{w_{1} \cdot \ldots \cdot w_{n+q}}=0$. But, by our choice of $w$,

$$
\liminf _{n \rightarrow \infty} \frac{w_{0} \cdot \ldots \cdot w_{-n+q+1}}{w_{1} \cdot \ldots \cdot w_{n+q}}=\lim _{n \rightarrow \infty} \frac{\beta^{n-q}}{\alpha^{n+q}}=\frac{1}{\alpha^{q} \beta^{q}} \lim _{n \rightarrow \infty}\left(\frac{\beta}{\alpha}\right)^{n}=0 .
$$

Thus, the operator $B_{w}$ is also supercyclic.

We saw in Theorem B that an invertible bilateral weighted shift can have the shadowing property without being expansive. The next result presents an additional condition which guarantees expansivity.

Proposition 39. Let $X=\ell_{p}(\mathbb{Z})(1 \leq p<\infty)$ or $X=c_{0}(\mathbb{Z})$, and consider a weight sequence $w=\left(w_{n}\right)_{n \in \mathbb{Z}}$ with $\inf _{n \in \mathbb{Z}}\left|w_{n}\right|>0$. If $F_{w}: X \rightarrow X$ has the shadowing property and the sequence $\left(n F_{w}^{n}\left(e_{0}\right)\right)_{n \in \mathbb{Z}}$ is not bounded, then $F_{w}$ is uniformly expansive.

Proof. By Proposition 29, it is enough to prove that $F_{w}$ is expansive. Assume that this is not the case. Then, by Theorem E(1),

$$
\sup _{n \in \mathbb{N}}\left|w_{1} \cdot \ldots \cdot w_{n}\right|<\infty \text { and } \sup _{n \in \mathbb{N}}\left|w_{-n} \cdot \ldots \cdot w_{-1}\right|^{-1}<\infty .
$$

Thus, the sequence $\left(z_{n}\right)_{n \in \mathbb{Z}}$ given by $z_{n}=F_{w}^{n+1}\left(e_{0}\right)(n \in \mathbb{Z})$ is bounded. Since $F_{w}$ has the shadowing property, Lemma 10 guarantees the existence of a bounded sequence $\left(y_{n}\right)_{n \in \mathbb{Z}}$ in $X$ such that $y_{n+1}=$ $F_{w}\left(y_{n}\right)+z_{n}$ for all $n \in \mathbb{Z}$. For each $n \in \mathbb{N}$, note that

$$
\begin{gathered}
y_{n}=F_{w}^{n}\left(y_{0}\right)+F_{w}^{n-1}\left(z_{0}\right)+\cdots+F_{w}\left(z_{n-2}\right)+z_{n-1}=F_{w}^{n}\left(y_{0}\right)+n F_{w}^{n}\left(e_{0}\right), \\
y_{-n}=F_{w}^{-n}\left(y_{0}\right)-F_{w}^{-n}\left(z_{-1}\right)-F_{w}^{-n+1}\left(z_{-2}\right)-\ldots-F_{w}^{-1}\left(z_{-n}\right)=F_{w}^{-n}\left(y_{0}\right)-n F_{w}^{-n}\left(e_{0}\right) .
\end{gathered}
$$

Write $y_{0}=\left(a_{n}\right)_{n \in \mathbb{Z}}$. Then

$$
\left\|y_{n}\right\| \geq\left|a_{0}+n\right|\left|w_{0} \cdot \ldots \cdot w_{n-1}\right| \quad \text { and } \quad\left\|y_{-n}\right\| \geq\left|a_{0}-n \| w_{-n} \cdot \ldots \cdot w_{-1}\right|^{-1}
$$

for every $n \in \mathbb{N}$. Since we are assuming that the sequence $\left(n F_{w}^{n}\left(e_{0}\right)\right)_{n \in \mathbb{Z}}$ is not bounded, these estimates imply that the sequence $\left(y_{n}\right)_{n \in \mathbb{Z}}$ is not bounded, a contradiction.

Proposition 40. Let $w=\left(w_{n}\right)_{n \in \mathbb{Z}}$ be a weight sequence with $\inf _{n \in \mathbb{Z}}\left|w_{n}\right|>0$. Assume that $F_{w}: \ell_{2}(\mathbb{Z}) \rightarrow \ell_{2}(\mathbb{Z})$ is hyponormal. If $\sigma_{p}\left(F_{w}^{*} F_{w}\right) \cap \mathbb{T}=\emptyset$, then $F_{w}$ is expansive.

Proof. Since $F_{w}\left(e_{n}\right)=w_{n} e_{n+1}$ and $F_{w}^{*}\left(e_{n}\right)=\overline{w_{n-1}} e_{n}, F_{w}^{*} F_{w}\left(e_{n}\right)=\left|w_{n}\right|^{2} e_{n}$, implying that

$$
\sigma_{p}\left(F_{w}^{*} F_{w}\right) \cap \mathbb{T}=\emptyset \Longleftrightarrow\left|w_{n}\right| \neq 1 \text { for all } n \in \mathbb{Z} .
$$

Since $F_{w}$ is hyponormal, it is well-known that the sequence $\left(\left|w_{n}\right|\right)_{n \in \mathbb{Z}}$ is increasing. Therefore, $\sup _{n \in \mathbb{N}} \mid w_{1}$. $\ldots \cdot w_{n} \mid=\infty$ if $\left|w_{0}\right|>1$, while $\sup _{n \in \mathbb{N}}\left|w_{-n} \cdot \ldots \cdot w_{-1}\right|^{-1}=\infty$ if $\left|w_{0}\right|<1$. Anyway, it follows from Theorem $\mathrm{E}(1)$ that $F_{w}$ is expansive.

\section{Remark 41.}

(a) We cannot remove the hyponormality hypothesis in Proposition 40. To see this, it is enough to choose $w$ so that $\left|w_{n}\right| \neq 1$ for all $n \in \mathbb{Z}, \sup _{n \in \mathbb{N}}\left|w_{1} \cdot \ldots \cdot w_{n}\right|<\infty$ and $\sup _{n \in \mathbb{N}}\left|w_{-n} \cdot \ldots \cdot w_{-1}\right|^{-1}<\infty$. Then, $\sigma_{p}\left(F_{w}^{*} F_{w}\right) \cap \mathbb{T}=\emptyset$ (by (14)), but $F_{w}$ is not expansive (by Theorem $\mathrm{E}(1)$ ). 
(b) The converse of the conclusion of Proposition 40 is not true in general. For instance, if $w=$ $\left(\ldots, \frac{1}{2}, \frac{1}{2}, \frac{1}{2}, 1,2,2,2, \ldots\right)$ then $F_{w}$ is hyponormal and uniformly expansive, but $\sigma_{p}\left(F_{w}^{*} F_{w}\right) \cap \mathbb{T} \neq \emptyset$.

\section{References}

[1] N. Aoki, K. Hiraide, Topological Theory of Dynamical Systems - Recent Advances, North-Holland, Amsterdam, 1994.

[2] S. Bartoll, F. Martinez-Gimenez, A. Peris, Operators with the specification property, J. Math. Anal. Appl. 436 (2016) 478-488.

[3] F. Bayart, É. Matheron, Dynamics of Linear Operators, Cambridge University Press, Cambridge, 2009.

[4] F. Bayart, I.Z. Ruzsa, Difference sets and frequently hypercyclic weighted shifts, Ergodic Theory Dynam. Systems 35 (3) (2015) 691-709.

[5] B. Beauzamy, Introduction to Operator Theory and Invariant Subspaces, North-Holland, Amsterdam, 1988.

[6] T. Bermúdez, A. Bonilla, F. Martínez-Giménez, A. Peris, Li-Yorke and distributionally chaotic operators, J. Math. Anal. Appl. 373 (1) (2011) 83-93.

[7] N.C. Bernardes Jr., A. Bonilla, V. Müller, A. Peris, Distributional chaos for linear operators, J. Funct. Anal. 265 (9) (2013) 2143-2163.

[8] N.C. Bernardes Jr., A. Bonilla, V. Müller, A. Peris, Li-Yorke chaos in linear dynamics, Ergodic Theory Dynam. Systems 35 (6) (2015) 1723-1745.

[9] J. Bès, Q. Menet, A. Peris, Y. Puig, Recurrence properties of hypercyclic operators, Math. Ann. 366 (1) (2016) 545-572.

[10] J. Bochi, Generic linear cocycles over a minimal base, Studia Math. 218 (2) (2013) 167-188.

[11] A. Bourhim, Bounded Point Evaluations and Local Spectral Theory, Dissertation, Trieste, 2000, arXiv:math/0008197v1.

[12] G. Costakis, M. Sambarino, Topologically mixing hypercyclic operators, Proc. Amer. Math. Soc. 132 (2) (2004) 385-389.

[13] D. Damanik, Lyapunov exponents and spectral analysis of ergodic Schrödinger operators: a survey of Kotani theory and its applications, in: Spectral Theory and Mathematical Physics: A Festschrift in Honor of Barry Simon's 60th Birthday, in: Proc. Sympos. Pure Math., vol. 76, Amer. Math. Soc., Providence, RI, 2007, pp. 539-563 (Part 2).

[14] H.R. Dowson, Spectral Theory of Linear Operators, Academic Press, London and New York, 1978.

[15] M. Eisenberg, Expansive automorphisms of finite dimensional vector spaces, Fund. Math. 59 (1966) $307-312$.

[16] M. Eisenberg, J.H. Hedlund, Expansive automorphisms of Banach spaces, Pacific J. Math. 34 (3) (1970) $647-656$.

[17] S. Grivaux, É. Matheron, Invariant measures for frequently hypercyclic operators, Adv. Math. 265 (2014) $371-427$.

[18] K.-G. Grosse-Erdmann, A. Peris Manguillot, Linear Chaos, Springer-Verlag, London, 2011.

[19] J.H. Hedlund, Expansive automorphisms of Banach spaces, II, Pacific J. Math. 36 (3) (1971) 671-675.

[20] A. Koropecki, E.R. Pujals, Some consequences of the shadowing property in low dimensions, Ergodic Theory Dynam. Systems 34 (4) (2014) 1273-1309.

[21] M. Mazur, Hyperbolicity, expansivity and shadowing for the class of normal operators, Funct. Differ. Equ. 7 (1-2) (2000) $147-156$.

[22] J. Ombach, The shadowing lemma in the linear case, Univ. Iagel. Acta Math. 31 (1994) 69-74.

[23] S.Yu. Pilyugin, Shadowing in Dynamical Systems, Lecture Notes in Mathematics, vol. 1706, Springer-Verlag, Berlin, 1999.

[24] S.Yu. Pilyugin, Spaces of Dynamical Systems, De Gruyter Studies in Mathematical Physics, vol. 3, De Gruyter, Berlin, 2012.

[25] S.Yu. Pilyugin, S. Tikhomirov, Lipschitz shadowing implies structural stability, Nonlinearity 23 (10) (2010) $2509-2515$.

[26] V.A. Pliss, Bounded solutions of nonhomogeneous linear systems of differential equations, in: Problems in the Asymptotic Theory of Nonlinear Oscillations, Nauk. Dumka, Kiev, 1977, pp. 168-173.

[27] G.T. Prǎjiturǎ, Irregular vectors of Hilbert space operators, J. Math. Anal. Appl. 354 (2) (2009) 689-697.

[28] C. Read, The invariant subspace problem for a class of Banach spaces, 2: hypercyclic operators, Israel J. Math. 63 (1) (1988) 1-40.

[29] H.N. Salas, Hypercyclic weighted shifts, Trans. Amer. Math. Soc. 347 (3) (1995) 993-1004.

[30] P. Walters, An Introduction to Ergodic Theory, Springer-Verlag, New York, 1982.

[31] K. Yosida, Functional Analysis, sixth edition, Springer-Verlag, Berlin and New York, 1980. 